\title{
EAUCTION WEB \& APP SYNCED SMART AGRICULTURE using IoT
}

\author{
S.Amudha, Jatin Nandwani, Akshay Agarwal, Navneeth Singh, Bharath Parihar
}

\begin{abstract}
Agribusiness is the most important process in our Nation. But nowadays, because of movement of individuals from provincial to urban there is prevention in agriculture. So to conquer this issue we go for shrewd horticulture procedures utilizing IoT. This undertaking incorporates different highlights like GPS based remote controlled checking, dampness and temperature detecting, gatecrashers startling, security, leaf wetness and legitimate water system offices. To ensure the safety of their financial transactions new crypto graphical algorithms was introduced already. We are making one such website and app for placing online auctions and providing an audience to them. The app has been designed using RESTful - API to sync its database with the website. And to encrypt the transactions we will be using SHA-256, a one way crypto-hash function, which only encrypts the data and doesn't decrypt it, making it a secure Hash Algorithm.
\end{abstract}

Keywords: Agriculture Monitoring,GSM SIM900 Module,Rainy Sensor, Arduino Uno microcontroller.

\section{I.INTRODUCTION}

The Gadgets that can be incorporated at any time anywhere in the world. So, there is no need to be present physically. So,this work is developing a website and App on E-Auction Synching their database using Restful-API. Providing security by SHA-256 (Secure hash algorithm), Google Analytic, Cloudfare.[1]. The objective of this project is to develop a system that allows a user to remotely control and monitor multiple PRODUCT ON-AUCTION using a cellular phone. This proposed system will be

a powerful and flexible tool that will offer this service at any time leading to overall cost reduction and energy saving.

The proposed approach for designing this system is to implement an online Auction and providing the security to our users by using SHA-256 Encrypt algorithm, Google Analytics, Cloud fare technology, Restful API.This will help us to track the Users and hackers, storing the confidential information in encrypted form And Sinking the database of our website and app which will help the user to use the same account and information for both Web and App.

Revised Manuscript Received on July 05, 2019.

S.Amudha, Software Engineering Department, SRM Institute of Science and Technology, Chennai ,India.

Jatin Nandwani, Software Engineering Department, SRM Institute of Science and Technology, Chennai, India.

Aksay Agarwal, Software Engineering Department, SRM Institute of Science and Technology, Chennai ,India.

Navneeth Sing and Bharat Parihar, Software Engineering Department, SRM Institute of Science and Technology, Chennai, India.

\section{WORKING PRINCIPLE}

In the operational segment, there are different types of sensors which are in the field, which includes sensors like. Temperature sensor/(LM32), dampness, and PIR sensor. All the above information's gathered from all these above sensors are eventually associated with the main module of microcontroller through RS232.In this control segment, all the obtained information is verified within the prescribed limitations esteems. The ON/OFF chances of every information surpasses the very limited esteem the signal, as and when its exchanged $\mathrm{ON}$, while the notifications are provided by the LED, which begins to flicker sequentially. The every alert is eventually senates a confirmation message further to the assigned agriculturist, while naturally the primary power is eventually turned OFF in a subsequent rate, to ensure periodic detecting. All these qualities are hence produced in its requisite site pages, which further transfers the received information to the assigned rancher who gets all the details of nitty gritty portrayal of all the different qualities.

In the manual mode, every client(s)does needs to turn ON/OFF the primary microcontroller henceforth by squeezing the very catch data into their own Android Application created for them. This henceforth gets finished with the assistance of the basic GSM Module help. In programmed mode, the main microcontroller can gets turned ON/OFF which does consequently alters the esteem surpass limit points. Not long after the very microcontroller is started, a basic, yet preliminary alarm must be then sent to the client in their system. This is henceforth accomplished in the end, by making an deeper impression through the very own GSM module.

Different types of parameters, which includes anomalies, like: Optimal Temperature ,Soil Mugginess, Overall soil dampness, and also the PIR sensors which demonstrates the very edge of the esteem and, also the water level sensor to show the water level inside the tank, or asset.

1. Download and Install the application from Google play store or open it in a browser to access the advantages..[5].

2. Signing can be done by using your Gmail account, Facebook account or by entering the details and verifying it by using the Phone Number.

3. The Information will be stored in our database which we have created by using PHP my admin and Mysqli quarries. 4. The password will be stored in 256 bit hash function (Encrypted form) by using SHA-256 (SHA- Secure Hash Algorithm)

5. Using of SHA-256, It allow only to encrypt, Decryption cannot be possible by admin too.

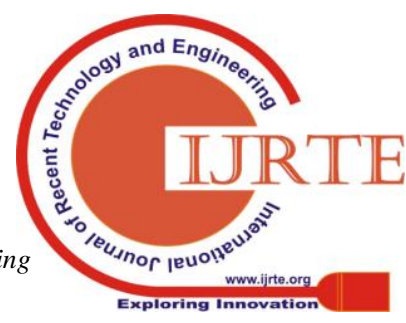


6. So it will keep the user information confidential.

7. Synchronization of database By Using RESTful-API

Which allow user to access the same account anytime from both Website and App.

8. By Using Google Analytics to track the user IP address, port number and Session for security propose.

9. Cloudfare which will act as a middleware, Using the name records and dns which won't allow any bot or hacker to penetrate into our websites and app. [2].

\section{SYSTEM ELABORATION} Server side and Client side. Ranch side sending is as appeared in figure1. It comprises of six techniques as takes after.

1. Sensing nearby rural parameters.

2. Identification of area of sensor and information gathering.

3 Transferring information from trim fields for basic leadership.

4.Decision help and early warming in light of information examination, space learning and history

created.

5. Actuation and control in view of choice.

6. Crop observing

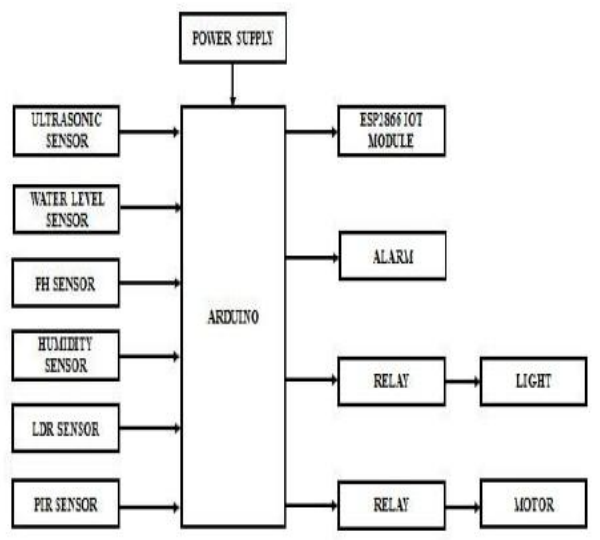

Figure 1: System Architecture

\section{A.GSM Module}

The GSM Modem can easily acknowledge any of the GSM systems, and arrange administrator SIM and it can easily act as a simply just like an ordinary cell phone, equipped with its own particular interesting yet unique telephone number. Its need to utilize this very function is it can as much utilize the RS-232 convention which also can be effectively associated with pairing of the controller. It can be utilized just like an ordinary telephone where it can be used to send and get SMS and make a call.

\section{B. PH Sensors}

A pH meter is one of the genuine instruments that is utilized to gauge the rate of hydrogen molecule change in encased water used plans, by displaying its general causticity, or the rate of alkalinity when passed on as the spurring power for $\mathrm{pH}$. The $\mathrm{pH}$ meter unquestionably measures the very refinement in electrical potential inspiration between the $\mathrm{pH}$ terminal and in addition a fundamental reference point as cathode, thusly influencing the $\mathrm{pH}$ to meter is as a less than
Proposed framework has three modules - Farm side,

dependable rule at last being derived as the "potentio metric $\mathrm{pH}$ meter". Its ability in the incredibly

electrical potential floats is effectively related to the rate of harming propensity or the respect $\mathrm{pH}$ of the game-plan. The $\mathrm{pH}$ meter is in like way used as a fundamental piece of the distinctive applications which are running from the lab experimentation to guarantee full quality control.

\section{Megunolink}

Associating through the connectively and linking with serial, remote or even an basic Ethernet, the MegunoLink has the ability to sends and also retrieve information to/from the Arduino. You can see the distinct crude information plot estimations on diagram maps shown below in the table.

MegunoLink can associate with any numerous types of Arduinos without a hassle. It sometimes affects by a

Moment's delay, or by catching and showing information from each and every one of them. This is a very extremely helpful tool for different utilities in remote ventures as should be obvious with what happens every one of their independent hubs.

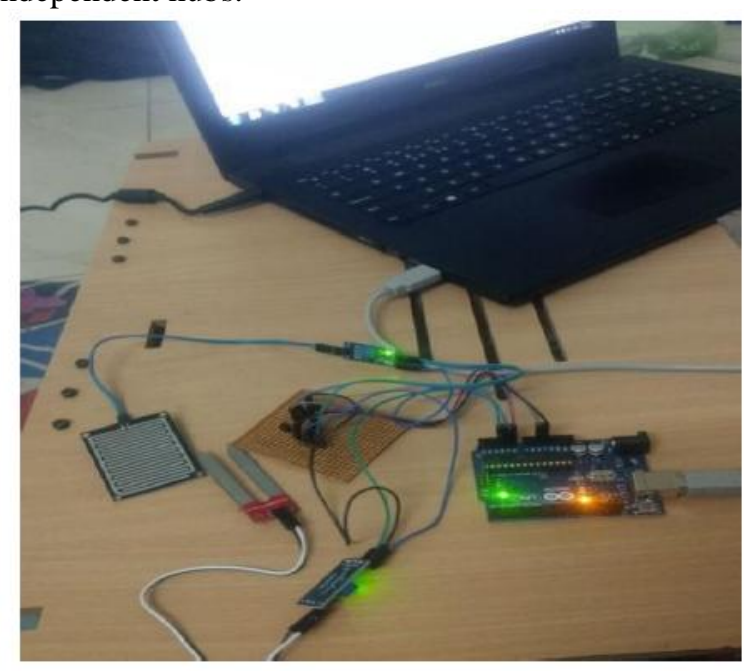

Figure 2: System Model

\section{RESTful-API}

A RESTful API is an application program interface (API) that uses HTTP requests to GET, PUT, POST and DELETE data. So, we are using RESTful-API to synch database of website and app. It will allow user to open the same account from an app and website both. This will ease the way to get notified about the increase of bidding and user won't have to open the website again and again or refreshing it to get notified.

\section{E. Cloudflare}

CloudFlare will act as a middleware to protect our website application from DDOS attacks. It won't allow any bot application to attack on our website or try to penetrate in our system. It is true that Cloud Flare uses CDN-like technology to act as a defender for our applications.

\section{F. AT command set}


The Remote activity control of the cell phone(GSM)operates with the help of SI Serial Interface (Information link of the Infrared Association), in which the $(\mathrm{AT}+\mathrm{C})$ does summons as indicated by the ETSI GSM 07.07, and the GSM 07.05 detail and in addition a few maker particular AT charges are accessible. In the modem system rule V.25 easily gets applied to the various grouping of the multiple interface charges. As indicated by this rule, orders should start specifically with the beginning of the character string "AT", and eventually will end with " $<\mathrm{CR}>$ " (= 0x0D). The overall utilization is judged by either "alright", or by "Blunder". A portion of selected summons utilized as a part of this task is recorded in Table 1.

\begin{tabular}{|c|l|l|}
\hline $\begin{array}{c}\text { Sr. } \\
\text { No. }\end{array}$ & Command & Descriptions \\
\hline I & AT+CGMI & Manufacturer Issue \\
& & ID code \\
\hline II & AT+CGMM & Issue model ID code \\
\hline III & AT+CSMS & Selecting msg \\
\hline IV & AT+(CMGR) & read SMS \\
\hline
\end{tabular}

Table 1: Commands and its Descriptions

\section{FUTURE WORK AND CONSLUSION}

This work successfully implemented app and web based on e-auction providing it with security benefits of SHA 256 and syncing the database of both website and application. For future improvements it can be upgraded by building up this framework for expansive sections of land of land. Likewise the framework can be coordinated to check the nature of the dirt and the development of product in each dirt. The sensors and

Microcontrollers are effectively interfaced with an established remote correspondence between all its different hubs. All the trial tests demonstrate that this is the very answer for different field exercises and upcoming water system issues. Usage of such different frameworks in this field can easily help to enhance the harvest yields. EAuction websites and app are beneficial for the upcoming generation because mobile is one of the mostly used communication device now a days. The user can access the application by sitting at any corner of the world at any time. Physical Presence would not be needed.

\section{ACKNOWLEDGMENT}

The authors would like to immensely thank theS oftware Engineering Department, SRM Institute of Science and Technology in Kattankulathur, Chennai for providing us with the opportunity of presenting this paper in International Conference and also for providing us with all of the necessary instruments used for the above experimental purpose. Its our great pleasure to thank our Guide for her immense support and their guidance to complete this project within the stipulated time schedule.

\section{REFERENCES}

[1] K.Lakshmisudha,SwathiHegde,Neha Kale and Shrutilyer, " Smart Precision Based Agriculture Using Sensors", International Journal of Computer Applications (0975-8887), Volume 146-No.11, July 2011.

[2] Nikesh Gondchawar,Dr.R.S.Kawitkar "IoT Based Smart Agriculture", International Journal of Advanced Research in Computer and Communication Engineering (IJARCCE), Vol.5, Issue 6, June 2016.

[3] M.K.Gayatri, J.Jayasakthi, Dr.G.S.Anandhamala, "Giving Smart Agriculture Solutions to Farmers for Better Yielding Using IoT", IEEE International Conference on Technological Innovations in ICT for Agriculture and Rural Development (TIAR 2015).

[4] ChetanDwarkani M,Ganesh Ram R,Jagannathan S, R. Priyatharshini,"Savvy Farming System Using Sensors for Agricultural Task Automation",IEEE International Conference on Technological Innovations in ICT for Agriculture and Rural Development (TIAR 2015).

[5] S.R.Nandurkar, V. R. Thool, R. C. Thool, "Outline and Development of Precision Agriculture System Using Wireless Sensor Network", IEEE International Conference on Automation, Control, Energy and Systems (ACES), 2014.

[6].Dr. V .VidyaDevi,G. MeenaKumari, "Constant Automation and Monitoring System for Modernized Agriculture" ,International Journal of Review and Research in Applied Sciences and Engineering (IJRRASE) Vol3 No.1. PP 7-12, 2013.

[7] Meonghun Lee, Jeonghwan Hwang, Hyun Yoe, "Horticultural Protection System Based on IoT", IEEE sixteenth International Conference on Computational Science and Engineering, 2013.

[8] Monika Jhuria, Ashwani Kumar, RushikeshBorse, "Picture Processing for Smart Farming: Detection of Disease and Fruit Grading", IEEE Second International Conference on Image Information Processing (ICIIP), 2013. [9] Orazio Mirabella and Michele Brischetto, "A Hybrid Wired/Wireless Networking Infrastructure for Greenhouse Management", IEEE Transactions on Instrumentation and Measurement, vol. 60, no. 2, pp 398-407, 2011.

[10] D.D.Chaudhary1, S.P.Nayse2, L.M.Waghmare, "Utilization of remote sensor systems for nursery parameter control in accuracy farming", International Journal of Wireless and Mobile Networks (IJWMN) Vol. 3, No. 1, February 2011.

[11] Q. Wang, A. Terzis and A. Szalay, "A Novel Soil Measuring Remote Sensor Network", IEEE Transactions on Instrumentation and Measurement, pp. 412-415, 2010.

\section{[12] DEXI WANG,YU IANG,FEI HE,MING} HU.,"Verification of

Implementations of Cryptographic Hash Function".,IEEE ACCESS 2017

[13] S.M.Sohan ,Craig Anslow, Frank Maurer,’SpyREST: Automated RESTful API Documentation Using an HTTP Proxy Server "., IEEE/ACM International Conference on Automated Software Engineering 2015

[14] Harris E. Michail,Apostolis Kotsiolis ,Athanasios Kakarountas ,George Athanasiou,Costas Goutisn.,"Hardware implementation of the Totally SelfChecking SHA-256 hash core".,IIEEE EUROCON $2015 \quad-\quad$ International 
Conference on Computer as a Tool (EUROCON) 2015

[15] Ram Krishna Dahal, Jagdish Bhatta,Tanka Nath Dahala.,"Performance Analysis Of SHA 2 and SHA 3".,International Journal on Cryptography and Information Security (IJCIS) 2013

\section{Authors Profiles}

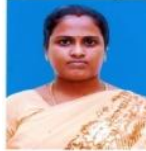

The first author of this paper work is S.Amudha ,working as assistant Professor in SRM Institute of Science and Technology,She completed her M.E in 2012 under AnnaUniversity of Technology,Chennai,Currently pursuing Ph.D in SRMIST.She has lots paper publication in Scopus index Journals of the various areas such as IoT,Wireless Sensor Networks, Wirelss Security,Bigdata Analytics,RFID etc.Her area of interest in Deep Learning,Data Analytics,SDN with IoT etc.She is member in professional society IET and IEI .

All other Authors in this work are U.G Students in SRM Institute of Science and Technology, Chennai in India. Their research area of interest include IoT,Big Data and Cloud Security. Based on their interest they selected their elective course in this area. They completed this work as for their final semester project work. 